\title{
Optimization of Nonthermal Fusion Power Consistent with Energy Channeling
}

\author{
P. B. Snyder, M. C. Herrmann, N. J. Fisch \\ Princeton Plasma Physics Laboratory \\ P. O. Box 451 \\ Princeton University, Princeton, New Jersey 08543
}

If the energy of charged fusion products can be diverted directly to fuel ions, non-Maxwellian fuel ion distributions and temperature differences between species will result. To determine the importance of these nonthermal effects, the fusion power density is optimized at constant- $\beta$ for nonthermal distributions that are self-consistently maintained by channeling of energy from charged fusion products. For D-T and D- ${ }^{3} \mathrm{He}$ reactors, with $75 \%$ of charged fusion product power diverted to fuel ions, temperature differences between electrons and ions increase the reactivity by $40-70 \%$, while nonMaxwellian fuel ion distributions and temperature differences between ionic species increase the reactivity by an additional $3-15 \%$.

Keywords

tokamak reactor, non-Maxwellian, alpha channeling, D-T reactor, D- ${ }^{3} \mathrm{He}$ reactor

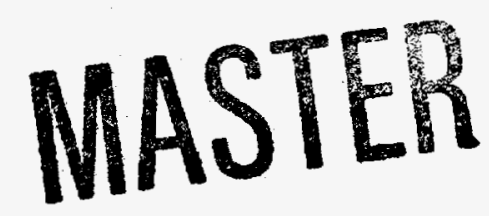




\section{Introduction}

There are advantages in operating a fusion reactor in regimes where the fuel ion temperature exceeds the electron temperature, ${ }^{1,2}$ i.e. in the "hot-ion mode." There are also potential advantages in operating in regimes where the fuel ion distribution is significantly non-Maxwellian. ${ }^{3-5}$ However, these regimes are difficult to realize. In typical $\mathrm{D}-\mathrm{T}$ fusion reactors, the alpha power, which heats the plasma, goes primarily to electrons, while ions and electrons lose energy at roughly the same rate. Thus, the electrons tend to be hotter than the ions. Furthermore, at densities and temperatures necessary for efficient D-T power production, the ion distributions tend to thermalize quickly, and so will generally be nearly Maxwellian.

The advantages of the hot-ion mode can, however, be realized if alpha power can be diverted directly to the fuel ions. Ions can then be hotter than electrons, especially in regimes where electron radiation losses are significant. In addition, non-Maxwellian features can be produced in the ion distribution because power might be absorbed preferentially by the fast tail of the ion distribution. Certain waves have been identified that might divert $\alpha$-power in this fashion, ${ }^{7,8}$ and a general analysis of the benefits of diverting $\alpha$-power by waves has been performed. ${ }^{6}$

The purpose of this paper is to further investigate the enhancement in fusion power that occurs when $\alpha$-particle power is diverted to fuel ions. In particular, we consider the effects of temperature differences between electrons and ions, temperature differences between ionic species, and non-Maxwellian ion distributions. We shall refer to non-Maxwellian ion distributions and temperature differences between species as "nonthermal effects." Note that these nonthermal effects all depend on the same conditions, i.e. on significant power diversion and relatively slow collisional equilibration. The nonthermal effects thus tend to occur simultaneously, and their effects on fusion power density tend to be multiplicative.

The paper is organized as follows. Each of the nonthermal effects will be briefly analyzed in Section II. In Section III the 0-dimensional energy balance equations will be modified to incorporate nonthermal effects. Section IV outlines a procedure for optimizing fusion power density at constant $\beta$. Sections $\mathrm{V}$ and VI present numerical results for $\mathrm{D}-\mathrm{T}$ and $\mathrm{D}-{ }^{3} \mathrm{He}$ reactors, respectively. Optimized, self-consistent operation points for archetype $\beta$-limited reactors 


\section{DISCLAIMER}

This report was prepared as an account of work sponsored by an agency of the United States Government. Neither the United States Government nor any agency thereof, nor any of their employees, make any warranty, express or implied, or assumes any legal liability or responsibility for the accuracy, completeness, or usefulness of any information, apparatus, product, or process disclosed, or represents that its use would not infringe privately owned rights. Reference herein to any specific commercial product, process, or service by trade name, trademark, manufacturer, or otherwise does not necessarily constitute or imply its endorsement, recommendation, or favoring by the United States Government or any agency thereof. The views and opinions of authors expressed herein do not necessarily state or reflect those of the United States Government or any agency thereof. 


\section{DISCLAIMER}

Portions of this document may be illegible in electronic image products. Images are produced from the best available original document. 
loosely based on the ARIES $\mathrm{I}^{9}$ and ARIES III $^{10}$ reactor designs will be presented. The contributions of each of the nonthermal effects will be analyzed separately. A brief conclusion appears in Section VII.

\section{Nonthermal Effects}

There are three nonthermal effects considered here. First, consider operation in the hot-ion mode $\left(T_{i}>T_{e}\right)$ which clearly leads to a large improvement in fusion power density at constant $\beta$, where $\beta$ is the ratio of particle pressure to magnetic energy density. For a given magnetic field, constant $\beta$ implies constant average plasma pressure $\langle p\rangle$. Ignoring impurities,

$$
\langle p\rangle=n_{i} T_{i}+n_{e} T_{e}=n_{i}\left(T_{i}+Z T_{e}\right),
$$

where $Z$ is the average charge state of the ions, $n_{i}$ and $n_{e}$ are the ion and electron number densities, and $T_{i}$ and $T_{e}$ are the ion and electron temperatures in energy units. For D-T, assuming a 50:50 D:T mix, the fusion power density $P_{f}$ is

$$
P_{f}=E_{f} n_{D} n_{T}\langle\sigma v\rangle=\frac{E_{f}\langle p\rangle^{2}\langle\sigma v\rangle}{4\left(T_{i}+T_{e}\right)^{2}},
$$

where $E_{f}$ is the energy released per fusion event, $n_{D}$ and $n_{T}$ are the deuteron and triton densities, and $\langle\sigma v\rangle$ is the fusion cross-section multiplied by relative velocity averaged over the two Maxwellian distributions at $T_{i}$. Because $\langle\sigma v\rangle$ is a function only of $T_{i}$, lowering $T_{e}$ at a given $T_{i}$ will always increase fusion power. For example, going from $T_{e}=T_{i}$ to $T_{e}=T_{i} / 2$ yields a $78 \%$ increase in fusion power density.

The second nonthermal effect considered involves temperature differences between ionic species. Alpha particle power will most likely be diverted to only one fuel ion species. Hence, it may be possible to maintain significant temperature differences between ion species, despite rapid ion-ion thermalization. This will, in some cases, increase the fusion power density at constant $\beta$. In general, $P_{f}$ will be optimized when the less massive ion species is hotter, except in cases where the more massive species is much less abundant than the less massive species. As an example, at 50:50 D:T, going from operation at $\left(T_{D}=T_{T}=15 \mathrm{keV}\right)$ to operation at $\left(T_{D}=16 \mathrm{keV}, T_{T}=14 \mathrm{keV}\right)$ will yield a $2.4 \%$ increase in fusion power. 
The third nonthermal effect considered involves non-Maxwellian features in the fuel ion distributions. A Maxwellian is not necessarily the distribution which optimizes $P_{f}$ at constant $\langle\dot{p}\rangle$. For example, in a D-T plasma with $T_{T}=T_{e}=15 \mathrm{keV}$, we find that the optimum deuterium distribution is a delta function near $75 \mathrm{keV}$, yielding a $75 \%$ increase in $P_{f}$ at constant $\langle p\rangle$ over a Maxwellian at $15 \mathrm{keV}$. At reasonable operating temperatures, ions in the tail of a Maxwellian contribute more fusion power per unit pressure than those in the bulk. A slowing-down distribution (SDD) is generally less peaked than a Maxwellian distribution, and therefore may produce more fusion power per unit pressure. Furthermore, if $\alpha$-particle power can be diverted to ions in such a way that the ions follow a diffusion path up to high energies, a slowing-down distribution of ions will result. This SDD will generally have higher fusion reactivity per pressure than the Maxwellian. Hence, if enough $\alpha$ power can be diverted to maintain a large SDD, overall fusion power might be significantly enhanced. In the following we shall refer to the fast non-Maxwellian feature of the ion distribution function as the SDD, which is in addition to the Maxwellian or bulk distribution.

\section{0-Dimensional Energy Balance}

When nonthermal effects are taken into account, energy balance in 0-D becomes somewhat more complicated. The two fuel ion species can now have different temperatures. Furthermore, in the D-T case, there are two slowingdown distributions, one for alphas and one for fast ions, each of which takes up pressure and gives up energy to each of the three bulk species.

First, consider the slowing-down distribution of $\alpha$-particles in 0-D. Alphas are created at an energy $E_{0 \alpha}$, and then collisionally slowed down. We assume the density of fast alphas and fast fuel ions to be small, so that interactions of these distributions with themselves and with each other can be ignored. The fast $\alpha$ distribution function $f_{\alpha}(\mathbf{v})$ then obeys the steady-state Fokker-Planck equation

$$
\nabla_{v} \cdot \mathbf{a} f_{\alpha}(\mathbf{v})=\frac{\left(1-f_{\text {div }}\right) P_{\alpha}}{4 \pi E_{0 \alpha} v_{0}^{2}} \delta\left(v-v_{0}\right),
$$

where $\mathbf{a}$ is the collisional deceleration of $\alpha$-particles, $P_{\alpha}$ is the portion of the fusion power density carried by alpha particles, and $f_{\text {div }}$ is the fraction of 
that power diverted directly to ions. Solving for $f_{\alpha}(v)$ yields

$$
f_{\alpha}(v)=\frac{\left(1-f_{\text {div }}\right) P_{\alpha}}{2 \pi v^{3} E_{0 \alpha} \nu_{E \alpha}}
$$

where $\nu_{E \alpha}=\nu_{E}^{\alpha / e}+\nu_{E}^{\alpha / D}+\nu_{E}^{\alpha / T}$, and where $\nu_{E}^{\alpha / x}$ is the rate of energy loss from alphas through collisions with species $x$. The functional dependence of $\nu$ on $E$ is given in Ref. 11. The pressure of the fast $\alpha$ distribution is then

$$
p_{\alpha} \equiv \frac{2}{3} \int_{0}^{v_{0}} \frac{1}{2} m_{\alpha} v^{2} f_{\alpha}(v) 4 \pi v^{2} d v=\frac{2}{3} \frac{\left(1-f_{\mathrm{div}}\right) P_{\alpha}}{E_{0 \alpha}} \int_{0}^{E_{0 \alpha}} \frac{d E}{\nu_{E \alpha}},
$$

and the fraction of $\alpha$ power given to species $x$ is

$$
f^{\alpha / x}=\frac{1}{E_{0 \alpha}} \int_{0}^{E_{0 \alpha}} \frac{\nu_{E}^{\alpha / x} d E}{\nu_{E \alpha}} .
$$

The treatment of the fast ion slowing-down distribution is analogous. The fast ions are considered to be drawn from a distribution with average ion energy $\bar{E}_{i}=3 T_{i} / 2$ and instantaneously accelerated to an energy $E_{0 s}$. The power input to this fuel ion slowing-down distribution is precisely the power diverted from $\alpha$-particles. Thus, to rewrite Eqs. 3-6 for fast ions, replace $\left(1-f_{\text {div }}\right) P_{\alpha}$ with $f_{\text {div }} P_{\alpha}$, and replace $E_{0 \alpha}$ in the denominator with $\left(E_{0 s}-\bar{E}_{i}\right)$. The subscript $s$ is used for slowing-down fuel ions. Hence, for example, Eq. 4 becomes

$$
f_{s}(v)=\frac{f_{\text {div }} P_{\alpha}}{2 \pi v^{3}\left(E_{0 s}-\bar{E}_{i}\right) \nu_{E s}} .
$$

The slowing-down ion density then becomes

$$
n_{s}=\int_{\bar{v}}^{v_{0}} f_{s}(v) 4 \pi v^{2} d v=\frac{f_{\mathrm{div}} P_{\alpha}}{\left(E_{0 s}-\bar{E}_{i}\right)} \int_{\bar{E}_{i}}^{E_{0 s}} \frac{d E}{\nu_{E s}} .
$$

For the case of a deuterium SDD reacting with a Maxwellian tritium distribution, the fusion power produced is

$$
P_{f s}=\int_{\bar{v}}^{v_{0}} f_{s}(v) E_{f} n_{T}\langle\sigma v\rangle_{\text {beam }} 4 \pi v^{2} d v=\frac{E_{f} n_{T} f_{\text {div }} P_{\alpha}}{\left(E_{0 s}-\bar{E}_{i}\right)} \int_{\bar{E}_{i}}^{E_{0 s}}\langle\sigma v\rangle_{\text {beam }} \frac{d E}{\nu_{E s} E},
$$


where $\langle\sigma v\rangle_{\text {beam }}$ is averaged over a tritium Maxwellian at $T_{T}$ and a deuterium beam at $E$.

In this model, some fraction of the alpha power is diverted to the ion SDD, while the rest is collisionally absorbed by the Maxwellian distributions of electrons, deuterons, and tritons. The slowing-down ion power is in turn given up to these bulk distributions. The bulk distributions also collisionally equilibrate and lose power through transport and radiation. The equations describing the $0-\mathrm{D}$ energy balance are thus

$$
\begin{aligned}
\frac{d E_{e}}{d t}= & \frac{3}{2} n_{e} \bar{\nu}_{E}^{e / D}\left(T_{D}-T_{e}\right)+\frac{3}{2} n_{e} \bar{\nu}_{E}^{e / T}\left(T_{T}-T_{e}\right)+f^{\alpha / e}\left(1-f_{\text {div }}\right) P_{\alpha} \\
& +f^{s / e} f_{\text {div }} P_{\alpha}-\frac{3}{2} n_{e} T_{e} / \tau_{E_{e}} \\
\frac{d E_{D}}{d t}= & \frac{3}{2} n_{D} \bar{\nu}_{E}^{D / e}\left(T_{e}-T_{D}\right)+\frac{3}{2} n_{D} \bar{\nu}_{E}^{D / T}\left(T_{T}-T_{D}\right)+f^{\alpha / D}\left(1-f_{\text {div }}\right) P_{\alpha} \\
& +f^{s / D} f_{\text {div }} P_{\alpha}-\frac{3}{2} n_{D} T_{D} / \tau_{E_{i}} \\
\frac{d E_{T}}{d t}= & \frac{3}{2} n_{T} \bar{\nu}_{E}^{T / e}\left(T_{e}-T_{T}\right)+\frac{3}{2} n_{T} \bar{\nu}_{E}^{T / D}\left(T_{D}-T_{T}\right)+f^{\alpha / T}\left(1-f_{\text {div }}\right) P_{\alpha} \\
& +f^{s / T} f_{\text {div }} P_{\alpha}-\frac{3}{2} n_{T} T_{T} / \tau_{E_{i}} \\
P_{\alpha}= & E_{\alpha} n_{D} n_{T}\langle\sigma v\rangle+\frac{E_{\alpha} n_{T} f_{\text {div }} P_{\alpha}}{\left(E_{0 s}-\bar{E}_{i}\right)} \int_{\bar{E}_{\mathbf{i}}}^{E_{0 s}}\langle\sigma v\rangle_{\text {beam }} \frac{d E}{\nu_{E} E}
\end{aligned}
$$

where $\bar{\nu}_{E}^{x / y}$ is rate of thermal equilibration of species $x$ with species $y$, and is proportional to $n_{y}$. Here $\tau_{E_{e}}$ and $\tau_{E_{i}}$ are total energy confinement times for electrons and ions, including both transport and radiative losses. Note also that $n_{D}$ and $n_{T}$ are the densities of the Maxwellian deuterons and tritons and do not include particles in the SDD's. The constant- $\beta$ constraint can be written

$$
\langle p\rangle=n_{e} T_{e}+n_{D} T_{D}+n_{T} T_{T}+p_{\alpha}+p_{s} .
$$

An analysis of the energy balance equations shows that, at any given set of temperatures, all terms save the loss term are proportional to $\langle p\rangle^{2}$. The loss term will go as $\langle p\rangle^{2}$ if $\tau_{E}$ goes as $1 / n$, which it does only approximately for empirical scaling laws. Nonetheless, as long as an ignited equilibrium can be reached near optimal operating temperatures, the power produced will be very nearly proportional to $\langle p\rangle^{2}$. Therefore, it is possible to extrapolate our 
results at a given value of $\langle p\rangle$ to other values of average pressure, so long as the pressure is sufficiently large to allow ignition near optimum operating temperatures.

\section{Optimization of a Nonthermal D-T Reactor}

We will now use further constraints to solve Eqs. 9-13 to find a selfconsistent operation point. We assume the relative concentration of deuterium and tritium is given. For the case of a deuterium SDD, a 50:50 D:T ratio implies $n_{D}+n_{s}=n_{T}$. In the following, we neglect impurities, including thermal alphas. The fast alpha density is found directly to be negligible. To implement the constraint on $\beta$, we choose a particular value for $\langle p\rangle$. We will use reactor parameters based on those chosen for ARIES I. ${ }^{9}$ In particular, we use an ARIES-like value of $\langle p\rangle=6.07 \times 10^{15} \mathrm{keV} / \mathrm{cm}^{3}$. Because ARIES I is envisioned to operate in $\mathrm{H}$-mode, we will use the ITER90H-P scaling law ${ }^{12}$ for $\tau_{E_{i}}$. Using other empirical H-mode scaling laws, or common L-mode scaling laws with appropriate enhancement factors, will affect the results very little.

However, $\tau_{E_{e}}$ will be treated differently. As mentioned, $\tau_{E_{e}}$ is a total energy confinement time for electrons, and thus must include losses due to bremsstrahlung, cyclotron, and line radiation, as well as transport losses. Furthermore, due to the benefits of hot-ion mode operation, it will turn out that power density is optimized by operating with $\tau_{E_{e}}$ values very near the minimum possible value for ignition, because the $T_{i} / T_{e}$ ratio is maximized when $\tau_{E_{i}} \gg \tau_{E_{e}}$. This optimal value of $\tau_{E_{e}}$ will, for all cases considered here, be lower than the value calculated using the transport scaling law with radiation losses. Note that, while it may not be possible to raise $\tau_{E_{e}}$ above the calculated value, it may be possible to lower it. Two possible mechanisms are reducing the wall reflectivity to increase synchrotron losses, and introducing high- $Z$ impurities to increase line radiation losses. It turns out to be possible to introduce enough very high-Z impurities to lower $\tau_{E_{e}}$ to its optimum value with little degradation in reactor performance. ${ }^{13}$ The degradation in fusion power density due to the small added pressure of the very high-Z impurities can generally be made small relative to the benefit of operating at an optimized $\tau_{E_{e}}$ value. Hence, in calculating an estimate of the nonthermal fusion power enhancement, we will treat $\tau_{E_{e}}$ as an adjustable parameter and 
optimize fusion power over it.

The three $0-D$ energy balance equations can now be numerically solved for the electron, deuteron, and triton temperatures at a given value of $\tau_{E_{e}}$. In cases where there is more than one ignited solution, the solution yielding higher $P_{f}$ will be used. The optimum value of $\tau_{E_{\epsilon}}$ can then be searched for, until an optimized self-consistent solution is found.

\section{D-T Numerical Results and Analysis}

The numerical calculations were performed using a parameter fit to the fusion cross section ${ }^{14}$ and analytic expressions for the various energy exchange rates. ${ }^{11}$ It should be noted that a number of approximations have been made. Impurities and profile effects have been ignored, for simplicity and because profiles cannot be calculated until the precise nature of the wave-particle interactions is known. The effects of $\alpha$-diversion on current drive, and the changes in bootstrap current at different operating points are also neglected. Current drive effects, including external power used to drive current, are not considered because, in cases where $\alpha$-particle power is diverted, profiles will be altered by the wave, and it is likely that the wave used to divert power to the ions may be used for current drive as well. ${ }^{8}$

Figures 1-4 exhibit the increasingly nonthermal behavior of the plasma as more $\alpha$-power is diverted to fuel ions. In all the figures, the operation point (i.e. the $\tau_{E_{e}}$ value) is chosen to optimize total fusion power density, not to optimize any particular nonthermal effect.

Figure 1 demonstrates the large increase in $T_{i} / T_{e}$ that can be obtained when significant amounts of $\alpha$-power are diverted. Similarly, Figure 2 exhibits the smaller increase in $T_{D} / T_{T}$ that is achieved when $\alpha$-power is diverted only to deuterons.

Figure 3 exhibits the contribution of the deuterium slowing-down distribution to the total fusion power, when $\alpha$-power is diverted to deuterons such that an SDD from $100 \mathrm{keV}$ down to the average ion energy is produced. When most $\alpha$-power is diverted, a substantial portion of the fusion power comes from non-Maxwellian ions. Of course, the net increase in fusion power due to the non-Maxwellian ions is more modest. The increase in fusion power is due only to the difference in reactivity per pressure between SDD ions and Maxwellian ions. The size of this increase in fusion power due to 
the SDD can be seen clearly by comparing the two lines in Fig. 4 . Figure 4 shows the overall increase in fusion power that is achieved when $\alpha$-power is diverted. This increase is due primarily to reduction of fast alpha pressure and operation in the hot-ion mode. The solid line shows the increase which occurs if $\alpha$-power is diverted to the tritium Maxwellian, while the dotted line shows the somewhat larger increase which occurs if power is diverted to a deuteron slowing-down distribution. The difference between the two lines is due largely to the increased reactivity per pressure of the deuterium SDD compared to a Maxwellian.

In Table I, we compare several operating points for D-T reactors, with and without $\alpha$-power diversion. The temperatures, densities, pressures and energy confinement times of the various species are given, along with the fusion power density produced in each case. The lower half of the chart separates the enhancements in $P_{f}$ over Case 1 into components due to ion temperature $\left(\left\langle\sigma v\left(T_{i}\right)\right\rangle / T_{i}^{2}\right.$ where $\left.T_{i}=\left(T_{D}+T_{T}\right) / 2\right)$, fast alpha pressure $\left(\left(\langle p\rangle-p_{\alpha}\right)^{2}\right)$, ion-electron temperature differences $\left(4 T_{i}^{2} /\left(T_{i}+T_{e}\right)^{2}\right)$, deuteron-triton temperature differences $\left(\left\langle\sigma v\left(T_{D}, T_{T}\right)\right\rangle /\left\langle\sigma v\left(T_{i}\right)\right\rangle\right)$, and slowing-down distribution effects. It is the last three enhancement factors that are of primary interest here.

Case 1 is a model of the operating point chosen in the ARIES I reactor study. It is used here as a reference point. The value of $\tau_{E_{e}}$ in this case is not optimized but rather is estimated based on transport and radiation. Because impurities and profile effects are not considered, some calculated values will vary significantly from ARIES I values.

In Case 2 there is still no diversion of $\alpha$-particle energy. However, fusion power density is optimized over $\tau_{E_{e}}$. This yields a substantial improvement in $P_{f}$, primarily due to the reduction in $\alpha$ pressure that accompanies operation at lower temperatures (alphas slow down much more quickly on cold electrons). Case 2 is provided so that the effects of $\alpha$-diversion can be analyzed independently of gains based only on optimization of $P_{f}$ over $\tau_{E_{e}}$. It should be noted, however, that the ARIES I operating regime was chosen for several reasons, including high current drive efficiency. Therefore, Case 2 may not be a preferred mode of operation due to the additional current drive expense in operating at low electron temperatures.

In Cases $3-5,75 \%$ of the $\alpha$-power is diverted directly to fuel ions. In Case 3 , the $\alpha$-power is diverted to the tritons in such a way that the tri- 
tium distribution remains approximately Maxwellian. This case exhibits the benefits of $\alpha$-power diversion in the absence of enhancements due to slowingdown ion distributions or desirable $T_{D} / T_{T}$ ratios. The benefits of diverting $\alpha$-power are quite apparent. The fusion power, $P_{f}$, is increased by a factor of 2.19 over Case 1 , and by a factor of 1.56 over Case 2. The improvement is due primarily to hot-ion mode operation and reduction in $\alpha$ pressure.

Case 4 is identical to Case 3 except that the $\alpha$-power is diverted to deuterium ions. This case exhibits the effect of differences between $T_{D}$ and $T_{T}$. Due to high deuteron-triton thermal equilibration rates, only a small temperature difference can be maintained between them. Hence there is only a slight additional increase in $P_{f}$, yielding a total enhancement factor of 2.22 over Case 1.

In Case 5 , the $\alpha$-power is diverted to a deuterium SDD with $E_{0 s}=$ $100 \mathrm{keV}$. This models the case where the wave absorbs energy from alphas and then damps on deuterons, moving the deuterons along a diffusion path out to an average energy of $100 \mathrm{keV}$ before they collisionally slow down to the average ion energy. While the reactivity of the SDD is significantly higher than that of the bulk, the improvement in $P_{f}$ is modest because only a low density slowing down distribution can be self-consistently maintained. The high density and low temperature of the bulk cause the ions in the slowing down distribution to lose energy very quickly, so that only a small number can be kept at high energies using diverted $\alpha$-power. In this case, a total $P_{f}$ enhancement of a factor of 2.25 over Case 1 is achieved.

The case in which $\alpha$-power is diverted to tritons, such that a tritium SDD is produced, is not presented, because it does not provide a significant improvement in $P_{f}$ over Case 3 . Hence, using a wave which damps on tritons will lead to a maximum enhancement factor of 2.19 . However, using a wave which damps on deuterons will produce a factor of at least 2.22 , and possibly as large as 2.25 if a non-Maxwellian distribution results. While this difference is relatively small, it might be enough to motivate the choice of a wave which damps on deuterons rather than tritons.

As stated, the operation point has been chosen to maximize $P_{f}$. Hence, Cases 3-5 do not represent the maximum achievable sizes of each nonthermal effect, but rather the maximum achievable total effect. In general, each of the nonthermal effects could be made larger in different regimes. For example, higher $T_{i} / T_{e}$ ratios can be achieved at higher temperatures, because the 
coupling between ions and electrons decreases. However, fusion reactivity decreases if $T_{i}$ exceeds the maximum of the $\langle\sigma v\rangle / T_{i}^{2}$ curve, and fast alpha pressure increases with $T_{e}$. Accounting for all three effects leads to an optimum operation point with $T_{i}$ somewhat above the maximum of the $\langle\sigma v\rangle / T_{i}^{2}$ curve, and $T_{e}$ somewhat below it.

\section{D- ${ }^{3} \mathrm{He}$ Numerical Results and Analysis}

Diverting charged fusion product power could be even more important for $\mathrm{D}^{3}{ }^{3} \mathrm{He}$ reactors, because large improvements over present tokamak performance appear necessary to burn $\mathrm{D}-{ }^{3} \mathrm{He}$. Furthermore, all of the fusion power is available as charged products. Hence more total power can potentially be diverted.

The model used for $\mathrm{D}-{ }^{3} \mathrm{He}$ is largely analogous to that used for D-T, except that both proton and alpha slowing-down distributions must be included. In addition, a scaling factor of 2.6 (an indication of the difficulties of igniting D- ${ }^{3} \mathrm{He}$ ) is added to the ITER90H-P scaling law to give $\tau_{E_{i}}$ values comparable to ARIES III values. The value $\langle p\rangle=3.427 \times 10^{16} \mathrm{keV} / \mathrm{cm}^{3}$ is also chosen to resemble the ARIES III design. This value is of course much larger than the value used for the D-T cases. Hence, the densities are higher despite the higher operating temperatures.

D- ${ }^{3} \mathrm{He}$ cases are presented in Table II. Case 1 models the ARIES III operating regime, in order to provide a reference point for the other cases. Here, the value of $\tau_{E_{e}}$ is not optimized, but rather estimated from transport and radiation. The fast alpha pressure is now rather small, due to high densities. However, the $14.7 \mathrm{MeV}$ protons take longer to slow down, and therefore occupy much more pressure. The ion and electron temperatures are nearly equal, with the larger collisional power input to electrons being offset by large electron radiative losses.

There is no good analogy to the D-T Case 2 because it is not possible to improve significantly upon Case 1 by lowering $\tau_{E_{e}}$. Case 2 in Table II shows the small benefits that could be achieved if $\tau_{E_{e}}$ could be increased. In Cases $3-5,75 \%$ of all fusion power is diverted to fuel ions. It should be noted that the dominant effect comes from diverting proton power, because at typical $\mathrm{D}-{ }^{3} \mathrm{He}$ parameters, alphas take up little pressure and slow down mostly on fuel ions without diversion. 
In Case 3, $75 \%$ of the fusion power is diverted to ${ }^{3} \mathrm{He}$, such that its distribution remains Maxwellian. Hence, Case 3 demonstrates the effect of diverting power in the absence of non-Maxwellian distributions or favorable $T_{D} / T_{3 \mathrm{He}}$ ratios. The factor of 1.92 increase in fusion power over Case 1 is due largely to hot-ion mode operation and reduction of fast proton pressure. The gains due to hot-ion mode operation are even greater than in D-T. High operating temperatures reduce the coupling between electrons and ions and allow very high $T_{i} / T_{e}$ ratios. However, gains from reducing fast fusion product pressure are smaller than in D-T. Comparison of Table II with Table I shows that the power enhancement in $\mathrm{D}-{ }^{3} \mathrm{He}$ is similar to that in $\mathrm{D}-\mathrm{T}$.

In Case 4, fusion product power is diverted to the deuterium Maxwellian rather than the ${ }^{3} \mathrm{He}$ Maxwellian. The direct improvement in $\langle\sigma v\rangle$ from having $T_{D}>T_{3 \mathrm{He}}$ is modest. However, note that the coupling between deuterons and electrons is smaller than the coupling between ${ }^{3} \mathrm{He}$ and electrons, due to the $Z^{2}$ factor in $\nu_{E}$. Hence, having $T_{D}>T_{3 H e}$ allows for a higher $T_{i} / T_{e}$ ratio and leads to a total $P_{f}$ enhancement factor of 2.00 over Case 1.

In Case 5, fusion product power is diverted to deuterons such that a deuterium slowing-down distribution extending up to $500 \mathrm{keV}$ is produced. This slowing-down distribution is larger than SDD's produced in D-T cases, and it yields a larger increase in $P_{f}$. The total fusion power in this case is enhanced by a factor of 2.08 over Case $1 . \mathrm{A}^{3} \mathrm{He}$ slowing-down distribution will not enhance $P_{f}$ over Case 3 . Hence diverting fusion product power to ${ }^{3} \mathrm{He}$ will produce a power enhancement factor of no more than 1.92 , while diverting to deuterons will lead to a $P_{f}$ enhancement factor of at least 2.00 , and possibly as large as 2.08 if a non-Maxwellian distribution is produced.

All three nonthermal effects have a larger impact in $\mathrm{D}^{3} \mathrm{He}$ cases than in $\mathrm{D}-\mathrm{T}$ cases because the higher operating temperatures reduce slowing-down rates and thermal equilibration rates.

\section{Conclusions}

We find that diverting $75 \%$ of the charged fusion product power to fuel ions roughly doubles the fusion power at constant- $\beta$ for both D-T and D${ }^{3} \mathrm{He}$ reactors. We have verified and refined this previously noted result ${ }^{6}$ in an analysis which treats nonthermal effects more precisely. In particular, we have included a slowing-down distribution of fast fuel ions, and we have 
allowed for separate temperatures for each fuel ion species. Furthermore, we have adopted a widely accepted empirical scaling law for energy confinement time. In addition, the operation points in this analysis are chosen to maximize total fusion power density. This selects a regime which obtains the maximum overall benefit from the numerous effects which enhance fusion power. The contributions of each effect can then be isolated and compared.

As expected, hot-ion mode operation generally provides the largest contribution to the enhancement in fusion power. With $75 \%$ of $\alpha$-power diverted in a $\mathrm{D}$ - $\mathrm{T}$ reactor, $T_{i} / T_{e}$ ratios of 1.5 are found. This translates into a fusion power enhancement factor of 1.4 over a case in which no $\alpha$-power is diverted. For $\mathrm{D}^{3} \mathrm{He}, T_{i} / T_{e}$ ratios around 1.6 and power enhancement factors as large as 1.7 are found when $75 \%$ of fusion power is diverted.

The second largest contribution to the fusion power enhancement arises from the reduction in pressure of the fast charged fusion products. This leads to a power enhancement factor of 1.4 for $\mathrm{D}-\mathrm{T}$, and 1.25 for $\mathrm{D}-{ }^{3} \mathrm{He}$, when $75 \%$ of charged fusion product power is diverted. These factors are of course multiplicative with those due to hot-ion mode.

Supplementing the two previously-studied sources of power enhancement mentioned above, are two additional contributions to the power enhancement which we have identified and analyzed. The first additional contribution is due to temperature differences between ion species. Diverting power to deuterons will lead to deuteron temperatures which are slightly higher than the temperatures of the other ions. Because of mass differences between ion species, this translates into an increase in fusion reactivity at constant beta. This increase is roughly $3 \%$ in D-T and $8 \%$ in $\mathrm{D}-{ }^{3} \mathrm{He}$, when $75 \%$ of charged fusion product power is diverted to deuterons.

The second additional contribution is provided by a fast fuel ion slowingdown distribution. Such a non-Maxwellian feature in the fuel ion distribution may be produced by preferential absorption of diverted power by the tail of the distribution. If such a deuterium slowing-down distribution is present, it can significantly enhance fusion power. A deuterium slowing-down distribution self-consistently created by the diversion of $75 \%$ of the charged fusion product power can increase fusion power by roughly an additional $3 \%$ in $\mathrm{D}-\mathrm{T}$ and $8 \%$ in $\mathrm{D}^{-}{ }^{3} \mathrm{He}$. Note that all of the increases mentioned above are multiplicative and can be attained simultaneously. Hence, for a D-T case where $75 \%$ of $\alpha$-power is diverted to deuterons, producing a slowing-down 
distribution, a total power enhancement factor of 2.25 can be attained.

In conclusion, the approximate factor of two improvement in fusion power density due to diversion of charged fusion product power has been verified in a more extensive numerical analysis. In addition, two new effects have been analyzed. These new effects can enhance fusion power density by an additional $5 \%$ in D-T and $15 \%$ in $\mathrm{D}^{3} \mathrm{He}$, in the cases considered here, where total fusion power density is optimized. These new effects will have an even greater impact in certain other regimes.

\section{Acknowledgments}

This work supported by DOE contract DE-AC02-76-CHO-3073, the National Science Foundation, and the Fannie and John Hertz Foundation. 


\section{References}

${ }^{1}$ J. G. Cordey and F. A. Haas, in Plasma Physics and Controlled Nuclear Fusion Research (Proc. 6th Int. Conf. on Plasma Phys. and Controlled Thermonuclear Fusion, Berchtesgaden, 1976) Vol. 2, IAEA, Vienna, 423 (1977).

${ }^{2}$ J. F. Clarke, Nucl. Fusion 20, 563 (1980).

${ }^{3}$ J. M. Dawson, H. P. Furth, and F. H. Tenney, Phys. Rev. Lett. 26, 1156 (1971).

${ }^{4}$ H. P. Furth and D. L Jassby, Phys. Rev. Lett. 32, 1976 (1972).

${ }^{5}$ D. L. Jassby, Nucl. Fusion 17, 309 (1977).

${ }^{6}$ N. J. Fisch and M. C. Herrmann, Princeton Plasma Physics Laboratory Report 2989 (to appear in Nucl. Fusion, 1994).

${ }^{7}$ N. J. Fisch and J. M. Rax, Phys. Rev. Lett. 69, 612 (1992).

${ }^{8}$ E. J. Valeo and N. J. Fisch, Phys. Rev. Lett. 73, 3536 (1994).

${ }^{9}$ R. W. Conn and F. Najmabadi, UCLA Report UCLA-PPG-1323 (1991).

${ }^{10}$ C. G. Bathke et al., LANL Report LA-UR-91-3171 (1991).

${ }^{11}$ D. L. Book, NRL Plasma Formulary (1990).

${ }^{12}$ J. P. Christiansen et al., Nucl. Fusion 32, 291 (1992).

${ }^{13}$ M. C. Herrmann, N .J Fisch, and P. B. Snyder, Bull. Am. Phys. Soc. 39, No. 7, 1759 (1994).

${ }^{14}$ H.-S. Bosch and G. M. Hale, Nucl. Fusion 32, 611 (1992). 
Figure Captions

\section{Figure 1}

Variation of ion and electron temperature with fraction of $\alpha$-power diverted to ions, when reactor operates in the regime which optimizes total fusion power density.

\section{Figure 2}

Variation of deuterium and tritium temperature with fraction of $\alpha$-power diverted to deuterons, when reactor operates in the regime which optimizes total fusion power density.

\section{Figure 3}

Increase in the fraction of fusion power produced by the nonthermal portion of the deuteron distribution when $\alpha$-power is diverted to a deuteron slowingdown distribution at $100 \mathrm{keV}$.

\section{Figure 4}

Increase in fusion power density with fraction of $\alpha$-power diverted to Maxwellian tritons (solid line) or to a slowing down distribution of deuterons (dotted line). The point at $\mathrm{x}=0$ corresponds to Case 2, the solid line at $\mathrm{x}=.75$ corresponds to Case 3 , and the dashed line at $\mathrm{x}=.75$ corresponds to Case 5 in Table I.

\section{Table Captions}

Table I

ARIES I-based D-T Reactor with 50:50 D:T ratio, ITER90H-P scaling for $\tau_{E_{i}},\langle p\rangle=6.07 \times 10^{15} \mathrm{keV} / \mathrm{cm}^{3}$.

\section{Table II}

ARIES III-based D- ${ }^{3} \mathrm{He}$ Reactor with 50:50 D: ${ }^{3} \mathrm{He}$ ratio, $2.6 \times$ ITER90H-P scaiing for $\tau_{E_{i}},\langle p\rangle=3.427 \times 10^{16} \mathrm{keV} / \mathrm{cm}^{3}$. 
Figure 1

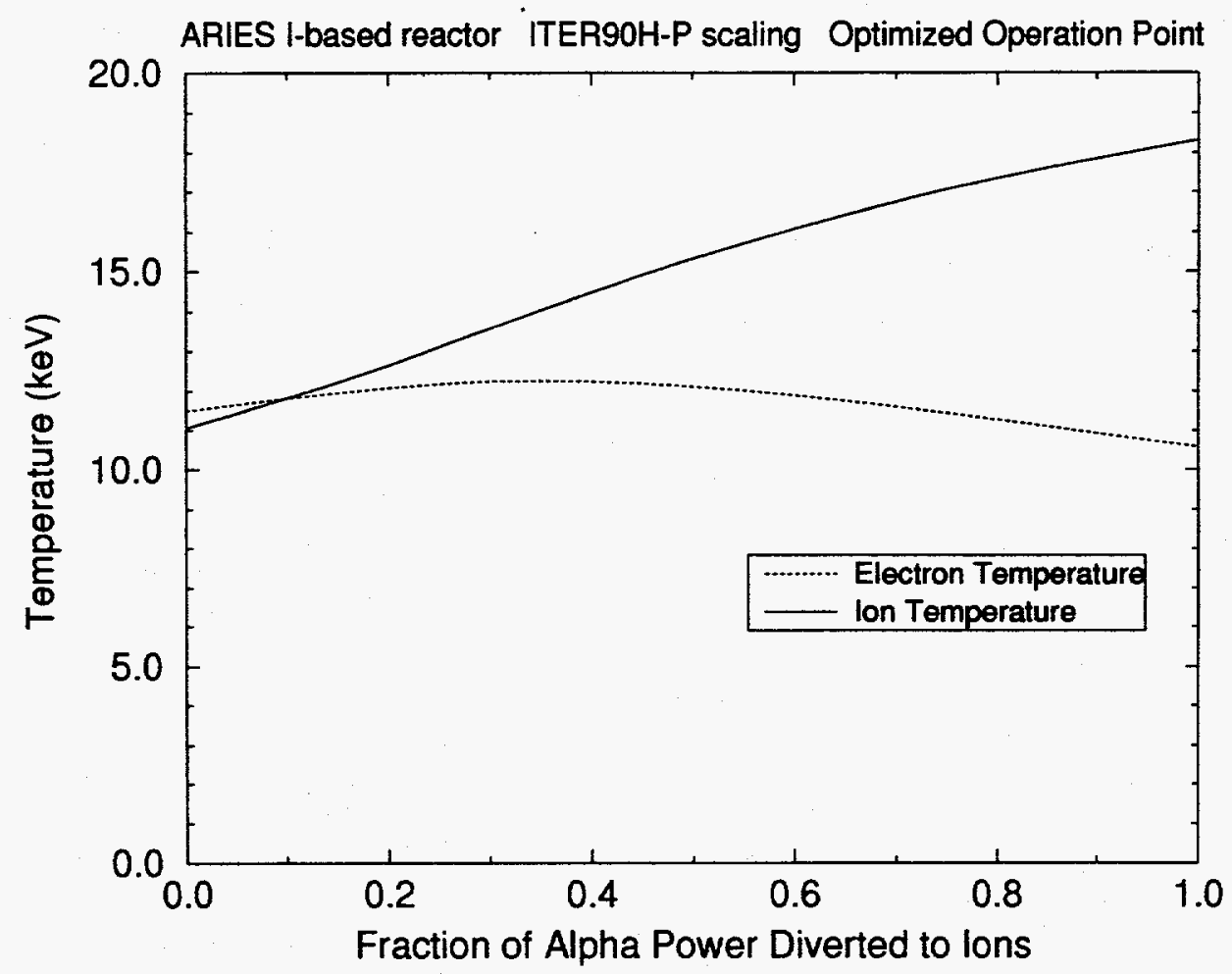


Figure 2

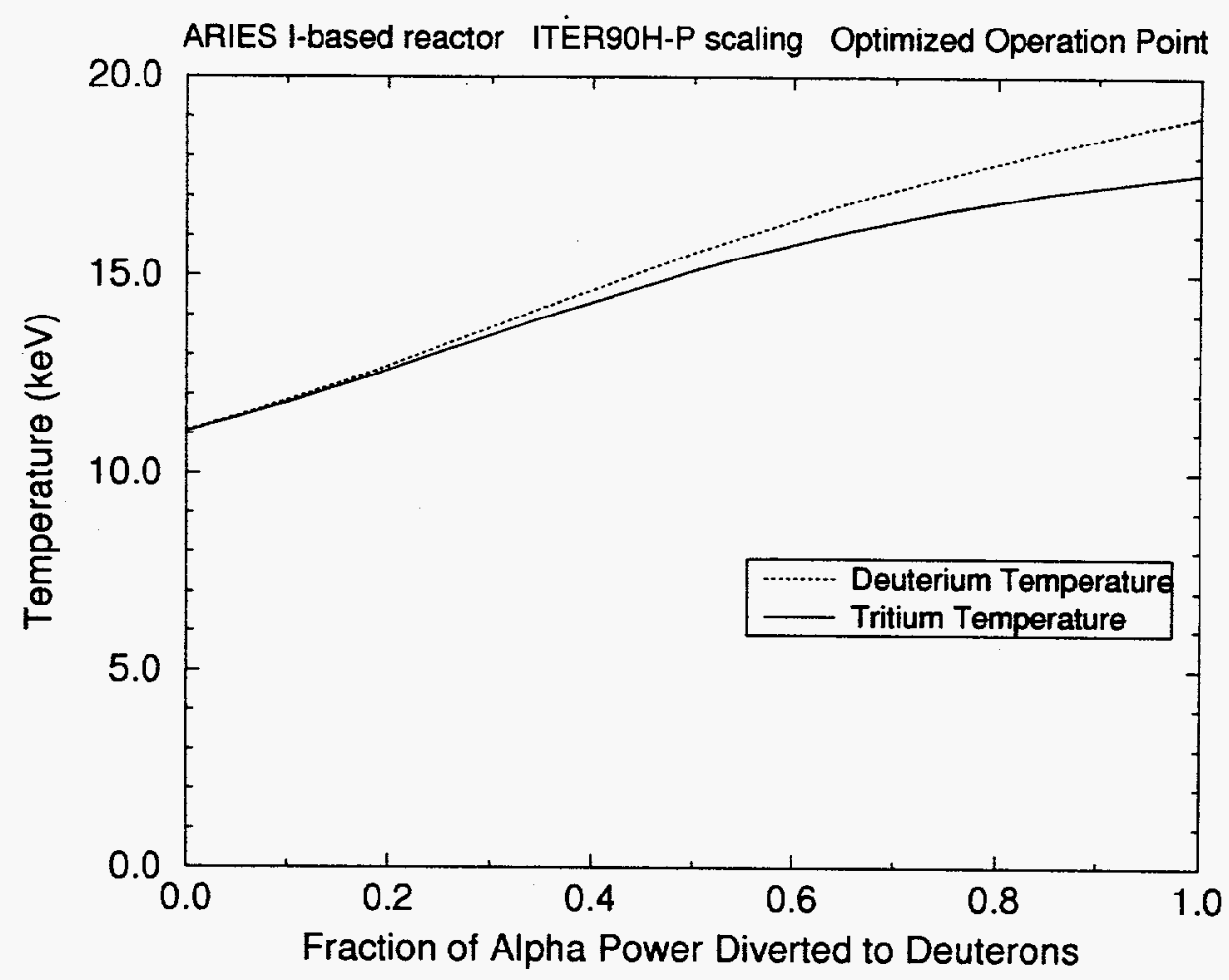


Figure 3

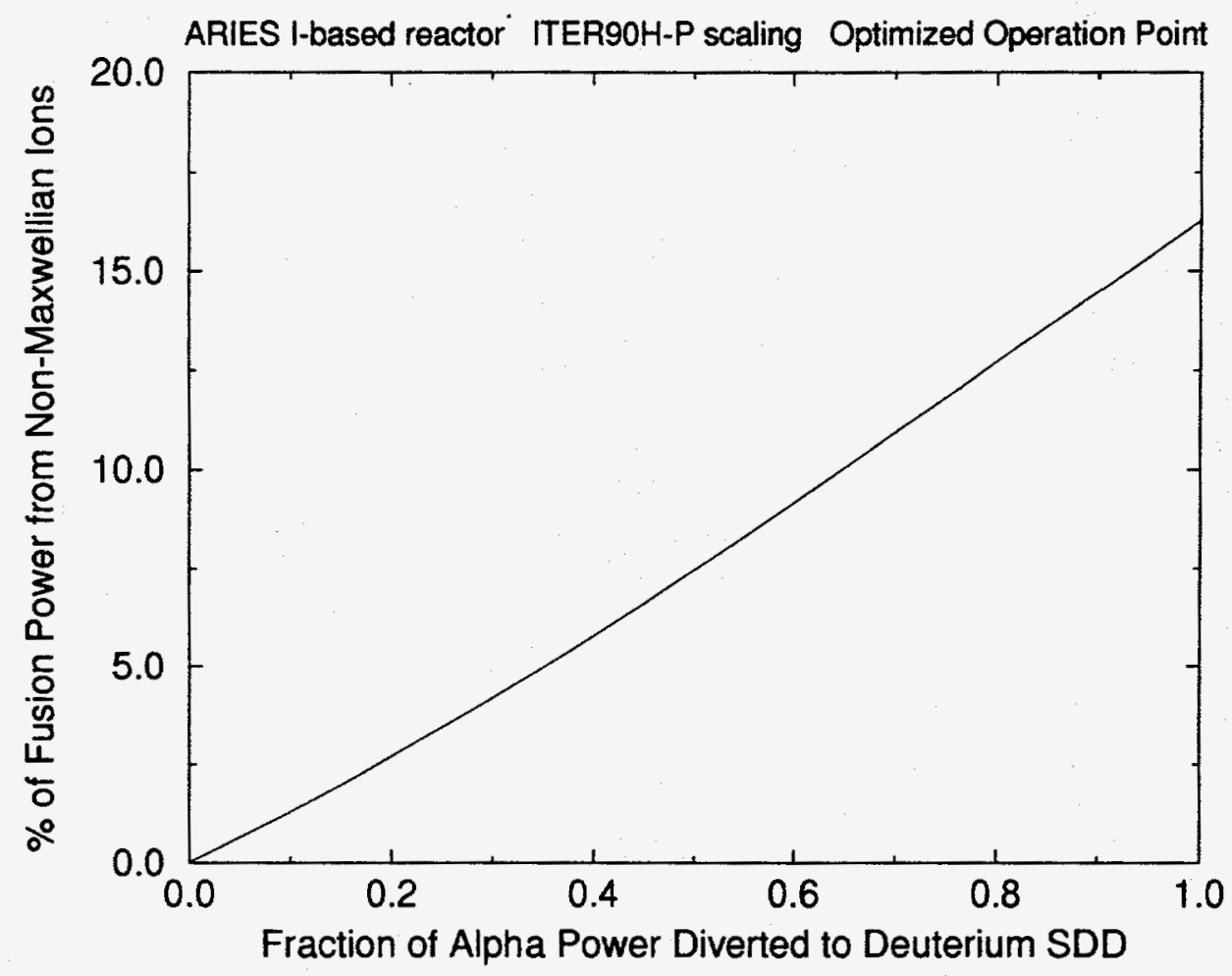


Figure 4

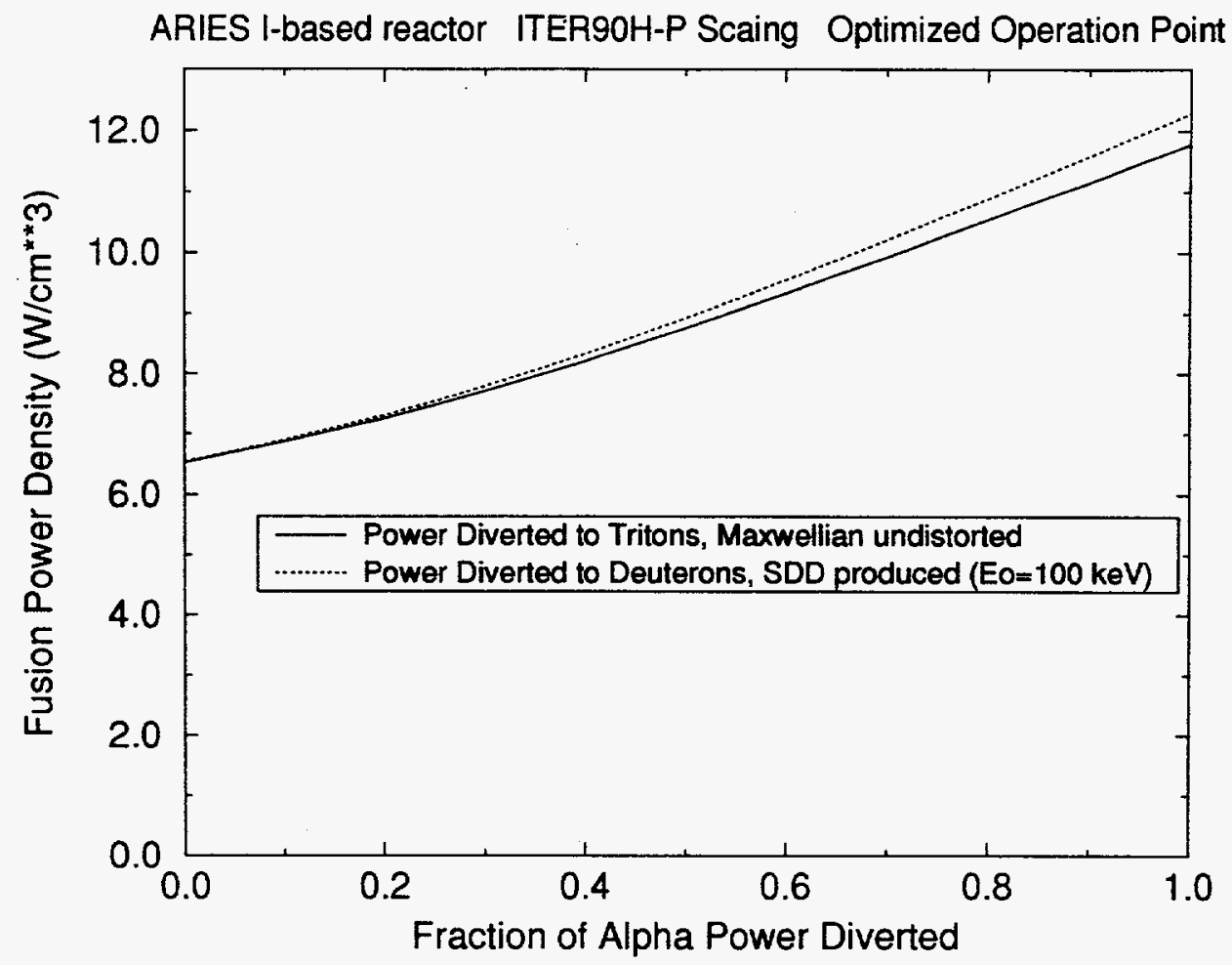


Table I

\begin{tabular}{|c|c|c|c|c|c|}
\hline Parameter & Case $1^{\circ}$ & Case 2 & Case 3 & Case 4 & Case 5 \\
\hline$T_{e}(k e V)$ & 20.0 & 11.4 & 11.4 & 11.5 & 11.4 \\
\hline$T_{D}(\mathrm{keV})$ & 20.1 & 11.0 & 16.4 & 17.5 & 16.6 \\
\hline$T_{T}(\mathrm{keV})$ & 19.9 & 11.0 & 17.5 & 16.6 & 16.6 \\
\hline$n_{e}\left(10^{14} / \mathrm{cm}^{3}\right)$ & 1.23 & 2.53 & 2.08 & 2.06 & 2.05 \\
\hline$n_{D}\left(10^{14} / \mathrm{cm}^{3}\right)$ & 0.62 & 1.26 & 1.04 & 1.03 & 0.97 \\
\hline$n_{T}\left(10^{14} / \mathrm{cm}^{3}\right)$ & 0.62 & 1.26 & 1.04 & 1.03 & 1.03 \\
\hline$n_{s}\left(10^{14} / \mathrm{cm}^{3}\right)$ & 0.0 & 0.0 & 0.0 & 0.0 & 0.05 \\
\hline$p_{e} /\langle p\rangle(\%)$ & 40.7 & 47.6 & 39.0 & 38.9 & 38.6 \\
\hline$p_{D} /\langle p\rangle(\%)$ & 20.4 & 23.0 & 28.0 & 29.7 & 26.7 \\
\hline$p_{T} /\langle p\rangle(\%)$ & 20.2 & 22.9 & 29.9 & 28.2 & 28.1 \\
\hline$p_{\alpha} /\langle p\rangle(\%)$ & 18.7 & 6.4 & 3.1 & 3.2 & 3.2 \\
\hline$p_{s} /\langle p\rangle(\%)$ & 0.0 & 0.0 & 0.0 & 0.0 & 3.5 \\
\hline$\tau_{E_{i}}(s)$ & 1.95 & 1.71 & 1.41 & 1.40 & 1.40 \\
\hline$\tau_{E_{e}}(s)$ & 0.95 & 0.77 & 0.40 & 0.39 & 0.37 \\
\hline$f_{\text {div }}$ & 0.0 & 0.0 & 0.75 & 0.75 & 0.75 \\
\hline$P_{f}\left(W / \mathrm{cm}^{3}\right)$ & 4.67 & 6.52 & 10.22 & 10.37 & 10.53 \\
\hline \multicolumn{6}{|c|}{$P_{f}$ Enhancement Factors Relative to Case 1} \\
\hline Total & 1.00 & 1.40 & 2.19 & 2.22 & 2.25 \\
\hline$T_{i}$ factor & 1.00 & 1.09 & 1.09 & 1.08 & 1.09 \\
\hline$p_{\alpha}$ factor & 1.00 & 1.33 & 1.42 & 1.42 & 1.42 \\
\hline$T_{i}>T_{e}$ factor & 1.00 & 0.96 & 1.43 & 1.43 & 1.41 \\
\hline$T_{D}>T_{T}$ factor & 1.00 & 1.00 & 0.99 & 1.01 & 1.00 \\
\hline SDD factor & 1.00 & 1.00 & 1.00 & 1.00 & 1.03 \\
\hline
\end{tabular}

Case 1: ARIES-I base operating point

Case 2: ARIES-I optimized over $\tau_{E_{\epsilon}}$

Case 3: $75 \%$ of $\alpha$ power diverted to tritium Maxwellian

Case 4: $75 \%$ of $\alpha$ power diverted to deuterium Maxwellian

Case 5: $75 \%$ of $\alpha$ power diverted to deuterium SDD ( $\bar{E}_{i}$ to $100 \mathrm{keV}$ ) 
Table II

\begin{tabular}{|c|c|c|c|c|c|}
\hline Parameter & Case 1 & Case 2 & Case 3 & Case 4 & Case 5 \\
\hline$T_{e}(\mathrm{keV})$ & 54.2 & 44.1 & 46.6 & 45.9 & 44.4 \\
\hline$T_{D}(\mathrm{keV})$ & 54.9 & 44.0 & 72.7 & 79.1 & 69.0 \\
\hline$T_{3_{\mathrm{He}}}(\mathrm{keV})$ & 55.1 & 44.0 & 75.2 & 73.3 & 69.5 \\
\hline$n_{e}\left(10^{14} / \mathrm{cm}^{3}\right)$ & 3.17 & 4.21 & 3.36 & 3.33 & 3.41 \\
\hline$n_{D}\left(10^{14} / \mathrm{cm}^{3}\right)$ & 1.06 & 1.40 & 1.12 & 1.12 & 1.04 \\
\hline$n_{3 \mathrm{He}}\left(10^{14} / \mathrm{cm}^{3}\right)$ & 1.06 & 1.40 & 1.12 & 1.12 & 1.14 \\
\hline$n_{s}\left(10^{14} / \mathrm{cm}^{3}\right)$ & 0.0 & 0.0 & 0.0 & 0.0 & 0.10 \\
\hline$p_{e} /\langle p\rangle(\%)$ & 50.1 & 54.2 & 45.7 & 44.6 & 44.2 \\
\hline$p_{D} /\langle p\rangle(\%)$ & 16.9 & 18.0 & 23.8 & 23.8 & 20.9 \\
\hline$p_{3 \mathrm{He}} /\langle p\rangle(\%)$ & 17.0 & 18.0 & 24.6 & 24.6 & 23.0 \\
\hline$p_{\alpha} /\langle p\rangle(\%)$ & 1.3 & 0.9 & 0.5 & 0.6 & 0.6 \\
\hline$p_{p} /\langle p\rangle(\%)$ & 14.7 & $\overline{8.8}$ & 5.4 & 5.6 & 5.4 \\
\hline$p_{s} /\langle p\rangle(\%)$ & 0.0 & 0.0 & 0.0 & 0.0 & 5.9 \\
\hline$\tau_{E_{i}}(s)$ & 7.20 & 7.00 & 5.30 & 5.19 & 5.10 \\
\hline$\tau_{E_{e}}(s)$ & 2.27 & 2.33 & 1.08 & 1.01 & 0.94 \\
\hline$f_{\text {div }}$ & 0.0 & 0.0 & 0.75 & 0.75 & 0.75 \\
\hline$P_{f}\left(W / \mathrm{cm}^{3}\right)$ & 2.21 & 2.34 & 4.24 & 4.42 & 4.59 \\
\hline \multicolumn{6}{|c|}{$P_{f}$ Enhancement Factors Relative to Case 1} \\
\hline Total & 1.00 & 1.06 & 1.92 & 2.00 & 2.08 \\
\hline$T_{i}$ factor & 1.00 & 0.94 & 0.95 & 0.94 & 0.97 \\
\hline$p_{\alpha}$ factor & 1.00 & 1.01 & 1.02 & 1.02 & 1.02 \\
\hline$p_{p}$ factor & 1.00 & 1.14 & 1.23 & 1.23 & 1.23 \\
\hline$T_{i}>T_{e}$ factor & 1.00 & 0.98 & 1.62 & 1.70 & 1.60 \\
\hline$T_{D}>T_{3_{H e}}$ factor & 1.00 & 1.00 & 0.99 & 1.01 & 1.00 \\
\hline SDD factor & 1.00 & 1.00 & 1.00 & 1.00 & 1.08 \\
\hline
\end{tabular}

Case 1: ARIES-III base operating point

Case 2: ARIES-III optimized over $\tau_{E_{e}}$

Case 3: $75 \%$ of fusion power diverted to ${ }^{3} \mathrm{He}$ Maxwellian

Case 4: $75 \%$ of fusion power diverted to deuterium Maxwellian

Case 5: $75 \%$ of fusion power diverted to deuterium $\operatorname{SDD}\left(\bar{E}_{i}\right.$ to $\left.500 \mathrm{keV}\right)$ 
Dr. F. Paoloni, Univ. of Wollongong, AUSTRALIA

Prof. R.C. Cross, Univ. of Sydney, AUSTRALIA

Plasma Research Lab., Australian Nat. Univ., AUSTRALIA

Prof. I.R. Jones, Flinders Univ, AUSTRALIA

Prof. F. Cap, Inst. for Theoretical Physics, AUSTRIA Prof. M. Heindler, Institut für Theoretische Physik, AUSTRIA

Prof. M. Goossens, Astronomisch Instituut, BELGIUM

Ecole Royale Militaire, Lab. de Phy. Plasmas, BELGIUM

Commission-Europoan, DG. XII-Fusion Prog., BELGIUM

Prof. R. Bouciqué, Rijksuniversiteit Gent, BELGIUM

Dr. P.H. Sakanaka, Instituto Fisica, BRAZIL

Prof. Dr. I.C. Nascimento, Instituto Fisica, Sao Paulo, BRAZIL Instituto Nacional De Pesquisas Espaciais-INPE, BRAZIL Documents Office. Atomic Energy of Canada Ltd., CANADA Ms. M. Morin, CCFMTTokamak de Varennes, CANADA Dr. M.P. Bachynski, MPB Technologies, Inc., CANADA

Dr. H.M. Skarsgard, Univ. of Saskatchewan, CANADA Prof. J. Teichmann, Univ. of Montreal, CANADA

Prof. S.R. Sreenivasan, Univ. of Calgary, CANADA

Prof. R. Marchand, INRS-Energie et Materiaux, CANADA

Dr. R. Bolton, Centre canadien de fusion magnétique, CANADA

Dr. C.R. James, Univ. of Alberta, CANADA

Dr. P. Lukác, Komenskeho Universzita, CZECHO-SLOVAKIA

The Librarian, Culham Laboratory, ENGLAND

Library, R61, Rutherford Appleton Laboratory, ENGLAND

Mrs. S.A. Hutchinson, JET Library, ENGLAND

Dr. S.C. Sharma, Univ. of South Pacific, FIJI ISLANDS

P. Măhönen, Univ. of Helsinki, FINLAND

Prof. M.N. Bussac, Ecole Polytechnique,, FRANCE

C. Mouttet, Lab. de Physique des Milieux lonisés, FRANCE

J. Radet, CEN/CADARACHE - Bat 506, FRANCE

Prof. E. Economou, Univ. of Crete, GREECE

Ms. C. Rinni, Univ. of loannina, GREECE

Preprint Library, Hungarian Academy of Sci., HUNGARY

Dr. B. DasGupta, Saha Inst. of Nuciear Physics, INDIA

Dr. P. Kaw, Inst. for Plasma Research, INDIA

Dr. P. Rosenau, Israel Inst. of Technology, ISRAEL

Librarian, International Center for Theo Physics, ITALY

Miss C. De Palo, Associazione EURATOM-ENEA, ITALY

Dr. G. Grosso, Istituto di Fisica del Plasma, ITALY

Prof. G. Rostangni, Istituto Gas lonizzati Del Cnr, ITALY
Dr. H. Yamato, Toshiba Res \& Devel Center, JAPAN

Prof. I. Kawakami, Hiroshima Univ., JAPAN

Prof. K. Nishikawa, Hiroshima Univ., JAPAN

Librarian, Naka Fusion Research Establishment, JAERI, JAPAN

Director, Japan Atomic Energy Research Inst., JAPAN

Prot. S. Itoh, Kyushu Univ., JAPAN

Research Info. Ctr., National Instit. for Fusion Science, JAPAN

Prof. S. Tanaka, Kyoto Univ., JAPAN

Library, Kyoto Univ., JAPAN

Prot. N. Inoue, Univ. of Tokyo, JAPAN

Secretary, Plasma Section, Electrotechnical Lab., JAPAN

Dr. O. Mitarai, Kumamoto Inst. of Technology, JAPAN

Dr. G.S. Lee, Korea Basic Sci. Ctr., KOREA

J. Hyeon-Sook, Korea Atomic Energy Research Inst., KOREA

D.I. Choi, The Korea Adv. Inst. of Sci. \& Tech., KOREA

Prof. B.S. Liley, Univ. of Waikato, NEW ZEALAND

Inst of Physics, Chinese Acad Sci PEOPLE'S REP. OF CHINA Librany, Inst. of Plasma Physics, PEOPLE'S REP. OF CHINA

Tsinghua Univ. Library. PEOPLE'S REPUBLIC OF CHINA

Z. Li, S.W. Inst Physics, PEOPLE'S REPUBLIC OF CHINA

Prof. J.A.C. Cabral, Instituto Superior Tecnico, PORTUGAL

Prof. M.A. Hellberg, Univ. of Natal, S. AFRICA

Prof. D.E. Kim, Pohang Inst. of Sci. \& Tech., SO. KOREA

Prof. C.I.E.M.A.T, Fusion Division Library, SPAIN

Dr. L. Stenflo, Univ. of UMEA, SWEDEN

Library, Royal Inst. of Technology, SWEDEN

Prof. H. Witheimson, Chalmers Univ. of Tech., SWEDEN

Centre Phys. Des Plasmas, Ecole Polytech, SWITZERLAND

Bibliotheek, Inst. Voor Plasma-Fysica, THE NETHERLANDS

Asst. Prof. Dr. S. Cakir, Middle East Tech. Univ., TURKEY

Dr. V.A. Glukhikh,Sci. Res. Inst. Electrophys.I Apparatus, USSR

Dr. D.D. Ryutov, Siberian Branch of Academy of Sci., USSR

Dr. G.A. Eliseev, I.V. Kurchatov Inst., USSR

Librarian, The Ukr.SSR Academy of Sciences, USSR

Dr. L.M. Kovrizhnykh, Inst. of General Physics, USSR

Kernforschungsanlage GmbH, Zentralbibliothek, W. GERMANY

Bibliothek, Inst. Für Plasmaforschung. W. GERMANY

Prof. K. Schindler, Ruhr-Universitát Bochum, W. GERMANY

Dr. F. Wagner, (ASDEX), Max-Planck-Institut, W. GERMANY

Librarian, Max-Planck-Institut, W. GERMANY 